\title{
Influence of Neoadjuvant Chemotherapy on Anastomotic Complications and Long-Term Prognosis after Laparoscopic Radical Gastric Cancer Surgery
}

\author{
Liu Chuanxu, Yao Zengwu, Jian Mi, Yu Bin, Cui Hongming, Zhao Yang, and Jiang Lixin* \\ The First Department of Gastrointestinal Surgery, Affiliated Yantai Yuhuangding Hospital of Qingdao University, Yantai 264000, China
}

*Corresponding authors: Jiang Lixin, The First Department of Gastrointestinal Surgery, Affiliated Yantai Yuhuangding Hospital of Qingdao University, Yantai 264000, China, Tel: 0535-6691999-83731; E-mail: jianglixin1969@hotmail.com

Received: 16 Jul, 2021 | Accepted: 19 Aug, 2021 | Published: 27 Aug, 2021

Citation: Chuanxu L, Zengwu Y, Mi J, Bin Y, Hongming C, et al. (2021) Influence of Neoadjuvant Chemotherapy on Anastomotic Complications and Long-Term Prognosis after Laparoscopic Radical Gastric Cancer Surgery. J Clin Case Stu 6(4): dx.doi.org/10.16966/2471-4925.232

Copyright: @ 2021 Chuanxu L, et al. This is an open-access article distributed under the terms of the Creative Commons Attribution License, which permits unrestricted use, distribution, and reproduction in any medium, provided the original author and source are credited.

\begin{abstract}
Objective: To analyze the occurrence of neoadjuvant chemotherapy on anastomotic complications after radical gastric cancer surgery and the influence of anastomotic complications on the long-term prognosis.

Methods: The clinical and pathological data of 1199 patients with gastric cancer who underwent radical gastrectomy in Yantai Yuhuangding Hospital Affiliated to Qingdao University from January 2010 to January 2020 were retrospectively analyzed, including anastomotic leakage, anastomotic stenosis and anastomotic bleeding. According to whether the patients had received neoadjuvant chemotherapy, they were divided into neoadjuvant chemotherapy group (170 cases) and non-neoadjuvant chemotherapy group (1029 cases). Objective to analyze the relationship between preoperative neoadjuvant chemotherapy and the incidence of anastomotic complications after radical gastrectomy and the impact of anastomotic complications on the prognosis of gastric cancer. After eliminating the potential confusion bias between the two groups by propensity score matching (PSM), the difference of incidence of anastomotic complications between the two groups and the relationship between anastomotic complications and longterm prognosis were compared.

Results: Before PSM, there was no significant difference in the incidence of anastomotic complications, including anastomotic leakage, anastomotic stenosis and anastomotic bleeding between neoadjuvant chemotherapy group and non-neoadjuvant chemotherapy group ( $P=0.732$ ). After PSM, there was no significant difference in the incidence of anastomotic complications either, including anastomotic leakage, anastomotic stenosis and anastomotic bleeding between neoadjuvant chemotherapy group and non-neoadjuvant chemotherapy group $(P=1.000)$. Binary logistic regression analysis showed that anemia and pneumonia were independent risk factors for anastomotic leakage after radical gastrectomy $(P<0.001)$, and neoadjuvant chemotherapy was not independent risk factor for anastomotic leakage after radical gastrectomy $(P=0.726)$. According to whether there were anastomotic complications, 1199 patients were divided into two groups. The survival curve was constructed, and the survival analysis was carried out. The results showed that the 5 -year survival rate of patients without anastomotic complications was better than that of patients with anastomotic complications (2.326 (1.570-3.448), $\mathrm{P}<0.001)$.
\end{abstract}

Conclusion: Neoadjuvant chemotherapy has no significant effect on the incidence of anastomotic complications after radical gastric cancer surgery, and the prognosis of patients with anastomotic complications is poor.

Keywords: Neoadjuvant chemotherapy; Radical gastrectomy; Anastomotic complications; Anastomotic leak; Anastomotic strictures; Anastomotic bleeding

\section{Introduction}

Although gastric cancer surgery has experienced decades of development, and with the advancement of science and technology, surgical methods have continued to innovate, but anastomotic complications are still one of the most serious complications after gastric cancer surgery, including anastomotic leakage, anastomotic stenosis and anastomotic bleeding, etc, which seriously affect the patient's postoperative recovery and long-term survival [1]. Laparoscopic surgery, as an emerging surgical method, not only causes less trauma to patients, but also has a lower incidence of postoperative complications than open surgery. It has been widely used in recent years [2]. Neoadjuvant chemotherapy can not only kill tumor cells, but also normal cells, reduce the body's resistance, and affect the patient's healing ability [3]. Whether neoadjuvant chemotherapy can affect the occurrence of anastomotic complications after laparoscopic gastric cancer surgery is still controversial. This study used propensity score matching method to analyze whether neoadjuvant chemotherapy is an independent risk factor for anastomotic complications after laparoscopic radical gastric cancer surgery. 


\section{Materials and Methods}

This study included 1,199 gastric cancer patients who underwent laparoscopic radical gastric cancer surgery at Yantai Yuhuangding Hospital, Qingdao University from 2011.01 to 2020.01. According to whether they accept neoadjuvant chemotherapy, they are divided into neoadjuvant chemotherapy group and non-neoadjuvant chemotherapy group. The neoadjuvant chemotherapy group enrolled 170 patients, including male: female 123: 47, age 36-82 years old. The non-neoadjuvant chemotherapy group included 1029 patients, including male:female 737:292, aged 23-87 years old.

\section{Inclusion criteria}

1). Primary gastric cancer, all underwent radical resection of gastric cancer; 2). Postoperative pathological examination confirmed gastric adenocarcinoma; 3 ). The clinicopathological data was complete and reliable. 4). All patients with neoadjuvant chemotherapy received XELOX regimen and XELOX neoadjuvant chemotherapy regimen. Carry out 2-3 cycles of neoadjuvant chemotherapy before surgery and radical gastric cancer surgery 4-6 weeks after the end of the chemotherapy, after excluding relevant surgical contraindications; 5). All patients started adjuvant chemotherapy within 8 weeks after the operation. The neoadjuvant chemotherapy group still uses the preoperative neoadjuvant chemotherapy to ensure that the perioperative chemotherapy reaches 8 cycles. The surgery group determines the specific chemotherapy program according to the postoperative pathological results.

\section{Exclusion criteria}

1). Patients who did not undergo radical surgery; 2). Serious underlying diseases; 3 ). Combined with malignant tumors of other systems; 4). Patients with incomplete clinicopathological data or lost to follow-up. 5). No XELOX program was given to patients with neoadjuvant chemotherapy; 6). Adjuvant chemotherapy was not given after operation.

\section{Collect the following data of patients}

1). General data: age, sex, height, weight; 2). Clinical pathological data: tumor location, degree of differentiation (refer to the $15^{\text {th }}$ edition of the Japanese Gastric Cancer Management Protocol), tumor diameter, Borrmann classification, Infiltration depth, lymph node metastasis, TNM staging, number of lymph nodes detected, vascular tumor thrombus, nerve infiltration, surgical procedures, postoperative chemotherapy, etc.; 3). Lifestyle: smoking history; 4). Combined with other diseases: Diabetes, hypertension, anemia and pneumonia.

The follow-up was conducted through telephone calls, text messages, and outpatient review. The follow-up period was as of January 2020, and the 5-year cumulative survival rate after surgery was calculated.

\section{Statistical analysis}

Use IBM SPSS 25.0 statistical software for statistical analysis. For non-matching samples, categorical variables are analyzed by $\chi^{2}$ test or Fisher exact probability method, and numerical variables are analyzed by independent sample $t$ test. For paired samples, McNemar's test is used to analyze categorical variables, and the paired sample $t$ test is used to analyze numerical variables. The Kaplan-Meier method was used to draw the survival curve (Figure 1), and the Log Rank test was used to evaluate whether the postoperative anastomotic complications had a significant impact on long-term survival. $\mathrm{P}<0.05$ indicates that the difference is statistically significant.

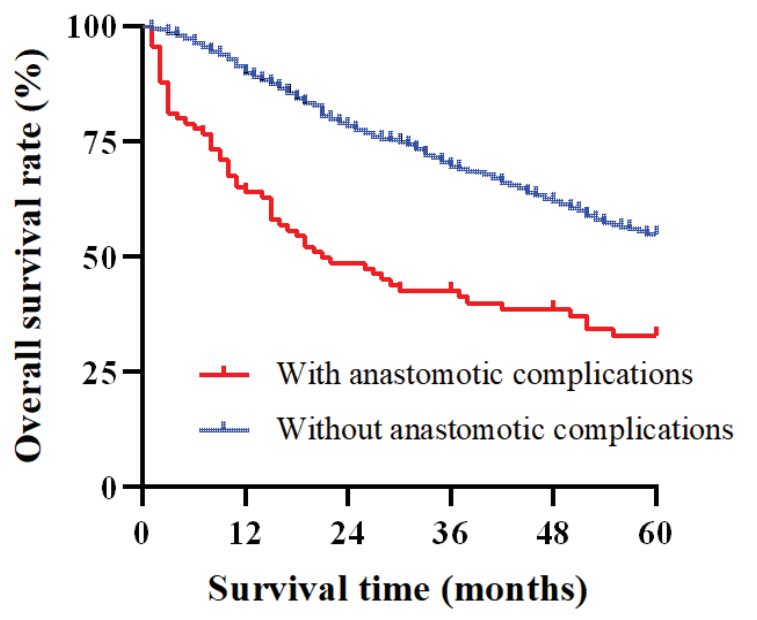

Figure 1: Compares the Kaplan-Meier curve of the overall survival rate of patients with and without anastomotic complications.

\section{Results}

Characteristics of various clinicopathological factors in neoadjuvant chemotherapy group and non-neoadjuvant chemotherapy group before and after PSM

A total of 1199 patients with gastric cancer were included in this study, of which 170 were in the neoadjuvant chemotherapy group and 1,029 were in the non-neoadjuvant chemotherapy group. Before applying PSM, there are significant differences between the neoadjuvant chemotherapy group and the non-neoadjuvant chemotherapy group in terms of age, BMI grade, tumor size, Borrmann classification, degree of differentiation, depth of tumor invasion, number of lymph node metastases, TNM stage, perineural invasion, venous cancerous embolus, tumor location, and Prevalence of pneumonia. After PSM, there is no significant difference in clinicopathological factors between the neoadjuvant chemotherapy group and the non-neoadjuvant chemotherapy group, which reduces various confounding factors and bias in the two groups, which is beneficial to the analysis of the impact of subsequent neoadjuvant chemotherapy on postoperative anastomotic complications (Table 1).

\section{Effect of neoadjuvant chemotherapy before and after PSM on postoperative anastomotic complications}

Before PSM, the incidence of anastomotic complications in the neoadjuvant chemotherapy group was about $8.2 \%$, including gastrointestinal anastomotic leakage (4.7\%), anastomotic stenosis (2.3\%), and anastomotic bleeding (1.2\%). The incidence of anastomotic complications in the non-neoadjuvant chemotherapy group was about $7.4 \%$, including gastrointestinal anastomotic leakage (4.2\%), anastomotic stenosis (2.4\%), and anastomotic bleeding ( $0.8 \%)$. There was no significant difference in the incidence of various anastomotic complications between the two groups. After PSM, the incidence of anastomotic complications in the neoadjuvant chemotherapy group was about 9.0\%, including gastrointestinal anastomotic leakage (5.3\%), anastomotic stenosis (3.0\%), and anastomotic bleeding (0.7\%). The incidence of anastomotic complications in the non-neoadjuvant chemotherapy group was about $9.0 \%$, including gastrointestinal 
Table 1: Demographic and clinical characteristics before and after propensity score matching.

\begin{tabular}{|c|c|c|c|c|c|c|}
\hline \multirow[b]{2}{*}{ Variables } & \multicolumn{3}{|c|}{ Before PSM } & \multicolumn{3}{|c|}{ After PSM } \\
\hline & NACT $(n=170)$ & $\begin{array}{l}\text { Non-NACT } \\
(n=1029)\end{array}$ & $\mathrm{P}$ & NACT $(n=132)$ & Non-NACT $(n=132)$ & $\mathbf{P}$ \\
\hline Male: Female & $123: 47$ & 737:292 & 0.845 & $98: 34$ & $97: 35$ & 1.000 \\
\hline Age & $62.82 \pm 8.595$ & $60.55 \pm 9.939$ & $0.005^{*}$ & $62.18 \pm 8.926$ & $60.43 \pm 9.373$ & 0.126 \\
\hline $\begin{array}{l}\text { BMI }\left(\mathrm{kg} / \mathrm{m}^{2}\right): \text { (Thin: Normal: Overweight: } \\
\text { Obese) }\end{array}$ & $4: 98: 44: 24$ & 41:502:371:115 & $0.032^{*}$ & $3: 74: 34: 21$ & $6: 73: 40: 13$ & 0.221 \\
\hline Tumor size $(\mathrm{cm})$ & $3.845 \pm 2.006$ & $4.995 \pm 2.678$ & $<0.001^{*}$ & $4.107 \pm 2.077$ & $4.337 \pm 2.186$ & 0.685 \\
\hline Smoking history (Yes: No) & $60: 110$ & $316: 713$ & 0.233 & $44: 88$ & $57: 75$ & 0.166 \\
\hline Diabetes (Positive: Negative) & 19:151 & 138:891 & 0.424 & 18:114 & 19:113 & 1.000 \\
\hline Anemia (Positive: Negative) & 9:161 & 60:969 & 0.781 & $8: 124$ & $17: 115$ & 0.093 \\
\hline Hypertension (Positive: Negative) & $37: 133$ & $176: 853$ & 0.141 & $30: 102$ & 33:99 & 0.775 \\
\hline Pneumonia (Positive: Negative) & $12: 158$ & $36: 993$ & $0.028^{*}$ & $8: 124$ & $8: 124$ & 1.000 \\
\hline $\begin{array}{l}\text { Borrmann classification } \\
\text { Early gastric cancer: type I: type II: type III: } \\
\text { type IV }\end{array}$ & $0: 16: 20: 116: 18$ & 149:57:8:738:77 & $<0.001^{*}$ & $14: 19: 89: 10$ & $13: 12: 96: 11$ & 0.461 \\
\hline Well differentiated: Poorly differentiated & $56: 114$ & $254: 775$ & $<0.023^{*}$ & $35: 97$ & $26: 106$ & 0.200 \\
\hline T stage T1:T2:T3:T4 & $0: 36: 80: 54$ & 147:133:90:659 & $<0.001^{*}$ & $0: 28: 62: 42$ & $0: 20: 59: 53$ & 0.088 \\
\hline N stage (N0:N1:N2:N3) & $12: 66: 73: 19$ & $355: 156: 263: 255$ & $<0.001^{*}$ & $12: 50: 56: 14$ & $13: 44: 54: 21$ & 0.303 \\
\hline TNM stage (I: II: III: IV) & $6: 75: 89: 0$ & 179:126:724:0 & $<0.001^{*}$ & $6: 54: 72: 0$ & $3: 53: 76: 0$ & 0.276 \\
\hline LVI(Positive: Negative) & $41: 129$ & 133:896 & $<0.001^{*}$ & $26: 106$ & $32: 100$ & 0.377 \\
\hline PNI (Positive: Negative) & $35: 135$ & $882: 147$ & $0.034^{*}$ & $17: 115$ & $21: 111$ & 0.584 \\
\hline $\begin{array}{l}\text { Tumor location (Antrum: Body: Fundus: } \\
\text { Complex site) }\end{array}$ & $104: 38: 21: 7$ & 657:209:48:115 & $<0.001^{*}$ & $81: 31: 15: 5$ & $85: 27: 6: 14$ & 0.851 \\
\hline $\begin{array}{l}\text { Operation method } \\
\text { Proximal gastrectomy: Total gastrectomy: } \\
\text { Distal gastrectomy }\end{array}$ & $14: 55: 101$ & $46: 321: 662$ & 0.093 & $4: 51: 77$ & $7: 52: 73$ & 0.457 \\
\hline
\end{tabular}

Note: the $8^{\text {th }}$ edition of TNM staging criteria for gastric cancer was used for pathological staging; PSM: Propensity Score Matching; NACT: Neoadjuvant Chemotherapy; LVI: Vascular Tumor Thrombus; PNI: Nerve Infiltration. BMI classification: Lean <18.5, normal: 18.5-23.9, overweight: 24-27.9, obesity: $\geq 28$; Tumor size is measured according to postoperative specimens; ${ }^{*} \mathrm{P}<0.05$, with statistical significance.

anastomotic leakage (7.5\%), anastomotic stenosis $(1.5 \%)$, and anastomotic bleeding $(0 \%)$. There was still no significant difference in the incidence of various anastomotic complications between the two groups. Before and after PSM and elimination of confounding factors, there was no significant difference in the incidence of postoperative anastomotic complications between the two groups, indicating that neoadjuvant chemotherapy is not an independent risk factor for anastomotic complications after radical gastric cancer (Table 2).

\section{Binary logistic regression analysis of influencing factors of anastomotic complications}

Before PSM, binary logistic regression analysis showed that the incidence of postoperative anastomotic complications in patients with anemia and pneumonia increased significantly. The remaining clinicopathological factors did not appear to be independent risk factors for anastomotic complications after gastric cancer surgery in the results of this study (Table 3 ).

\section{Influence of postoperative anastomotic complications before PSM on prognosis}

According to the presence or absence of anastomotic complications, 1199 patients with gastric cancer were divided into 2 groups, followed up to collect the 5-year survival data of these patients, construct a survival curve and conduct survival analysis. The results showed that patients without anastomotic complications had a better prognosis than patients with anastomotic complications. Therefore, it is very important to study the influencing factors of anastomotic complications. HR $(95 \% \mathrm{CI})=2.326(1.570-3.448) \mathrm{P}<0.001 \quad(\log$ rank test).

\section{Discussion and Conclusion}

Gastric cancer is one of the most common malignant tumors and the fifth most common malignant tumor in the world. It accounts for the third cause of tumor death and endangers human health [4]. Many patients often have no obvious symptoms in the early stage of gastric cancer, and most of them are already in the middle and late stages of the disease when they see a doctor. Preoperative neoadjuvant chemotherapy can shrink tumors, reduce tumor staging, and increase the $\mathrm{R}_{0}$ resection rate of surgery [5] more and more patients receive neoadjuvant chemotherapy and benefit from it. However, neoadjuvant chemotherapy is a general double-edged sword. While destroying tumor cells, it can also damage normal cells and tissues, leading to low immunity and a series of complications. The main treatment for gastric cancer is surgical resection, and its prognosis is related to potentially serious complications, such as anastomotic leakage, anastomotic stenosis, and anastomotic bleeding. These anastomotic complications not only reduce the patient's quality of life and bring painful treatment experience to the patient, but also seriously affect the patient's postoperative recovery and even endanger the patient's life, resulting in a poor prognosis [6]. It has been clear that the 
Table 2: Effect of neoadjuvant chemotherapy on postoperative complications before and after propensity score.

\begin{tabular}{|l|c|c|c|c|c|c|}
\hline \multirow{2}{*}{ Variables } & \multicolumn{3}{|c|}{ Before PSM } & \multicolumn{3}{c|}{ After PSM } \\
\cline { 2 - 7 } & $\begin{array}{c}\text { NACT } \\
\text { (n=170) }\end{array}$ & $\begin{array}{c}\text { Non-NACT } \\
\text { (n=1029) }\end{array}$ & $\mathbf{P}$ & $\begin{array}{c}\text { NACT } \\
\text { (n=132) }\end{array}$ & $\begin{array}{c}\text { Non-NACT } \\
\text { (n=132) }\end{array}$ & $\mathbf{P}$ \\
\hline $\begin{array}{l}\text { Anastomotic } \\
\text { complications }\end{array}$ & 14 & 77 & 0.732 & 12 & 12 & 1.000 \\
\hline $\begin{array}{l}\text { Anastomotic } \\
\text { leak }\end{array}$ & 8 & 43 & 0.752 & 7 & 10 & 0.581 \\
\hline $\begin{array}{l}\text { Anastomotic } \\
\text { stenosis }\end{array}$ & 4 & 25 & 1.000 & 4 & 2 & 0.688 \\
\hline $\begin{array}{l}\text { Anastomotic } \\
\text { bleeding }\end{array}$ & 2 & 9 & 1.000 & 1 & 0 & 1.000 \\
\hline
\end{tabular}

Note: PSM: Propensity Score Matching; NACT: Neoadjuvant Chemotherapy.

Table 3: Binary logistic regression analysis of influencing factors of anastomotic complications in 1199 patients with gastric cancer.

\begin{tabular}{|l|c|c|}
\hline \multicolumn{1}{|c|}{ Variables } & OR (95\% Cl) & P \\
\hline Anemia & & $\mathrm{P}<0.001^{*}$ \\
\hline No & 1 & \\
\hline Yes & $4.129(2.083-8.186)$ & \\
\hline Pneumonia & & $\mathrm{P}<0.001^{*}$ \\
\hline No & 1 & \\
\hline Yes & $4.014(1.835-8.778)$ & \\
\hline
\end{tabular}

Note: ${ }^{*}$ means $\mathrm{P}<0.05$, which is statistically significant.

occurrence of anastomotic complications is related to the patient's own factors and external factors. Self-factors include whether you have diabetes, hypoproteinemia, anemia, pneumonia, high blood pressure, long-term smoking history, application of anti-angiogenesis targeted drug therapy, etc. External factors include the surgical method of gastrointestinal reconstruction, the tension of anastomotic sutures, and the proficiency of the surgeon. However, the relationship between neoadjuvant chemotherapy and postoperative anastomotic complications is controversial. This study did not find that neoadjuvant chemotherapy was associated with the incidence of anastomotic complications (including anastomotic leakage, anastomotic stenosis, and anastomotic bleeding) after gastric cancer surgery before and after the propensity score matching. Moreover, the binary logistic regression analysis did not find that neoadjuvant chemotherapy was an independent risk factor for anastomotic complications.

The incidence of anastomotic leakage is about 2.1-14.6\%, and the mortality rate is as high as $50 \%$. It is an independent predictor of poor survival for patients with gastric cancer. Its treatment methods include drainage, nutritional support, and prevention of severe sepsis or consideration of a second operation when conservative treatment fails, but there is still no very effective treatment method [7-9]. At present, it is generally believed that the three main factors that affect the occurrence of anastomotic leakage after gastric cancer are poor anastomotic blood supply, excessive anastomotic tension, and poor local condition of the intestine at the anastomosis. Anastomotic blood supply is related to many factors, including diabetes, anemia, malnutrition, long-term smoking, anti-vascular drugs, etc., which can lead to anastomotic leakage due to insufficient blood supply to the microcirculation of the anastomosis. The tension of the anastomosis is related to the length of the free intestine during the operation and the operation method. The condition of the intestinal tube at the anastomosis may be related to whether there is intestinal obstruction, nutritional status, and whether to undergo neoadjuvant chemotherapy. It can be seen that neoadjuvant chemotherapy may be a risk factor for anastomotic leakage. It may affect the occurrence of anastomotic leakage by affecting the local tissue healing ability of the anastomosis and the supply of microcirculation blood $[10,11]$. However, at present, many centers have shown through research that whether neoadjuvant chemotherapy is performed has nothing to do with the risk of anastomotic leakage $[12,13]$. Neoadjuvant chemotherapy will reduce the tumor volume, increase the $\mathrm{R}_{0}$ resection rate of surgery, preserve more intestines, reduce the tension of the anastomosis, and facilitate the healing of the anastomosis. The study of Maggiori, et al. found that complete pathological remission after neoadjuvant chemotherapy would reduce the incidence of postoperative complications related to anastomotic leakage or infection [14]. With the continuous advent of new chemotherapeutics, the current accuracy of neoadjuvant chemotherapy drugs is higher, and the side effects brought by them are gradually reduced. Moreover, tumor shrinkage will offset the difficulty of surgery caused by the larger tumor volume to some extent, which may also be one of the reasons why the incidence of anastomotic leakage has not increased.

Anastomotic stenosis is mostly caused by postoperative anastomotic tissue edema and inflammation leading to tissue hyperplasia or scar contracture, which eventually leads to gastrointestinal obstruction, nutritional disorders, and then affects the patient's postoperative recovery. At present, it is believed that the main reason for the anastomotic stenosis is the width of the gastric tube after the operation and the way the anastomosis is sutured $[15,16]$. There is no clear conclusion about the impact of neoadjuvant on anastomotic stenosis. Zhu. et al. through univariate and multivariate analysis, neoadjuvant chemotherapy is not an independent risk factor for anastomotic stenosis [15]. This is the same as the results of this study. Neoadjuvant chemotherapy may cause tissue edema, decreased immunity of the body, and proliferation of inflammatory tissues [10]. However, it reduces the tumor, which is conducive to better progress of surgery, and its effect on anastomotic stenosis is far inferior to surgery and suture methods.

Postoperative anastomotic bleeding is an uncommon but potentially life-threatening complication. Strict surgical procedures are essential to prevent postoperative bleeding. Most current studies believe that postoperative anastomotic bleeding is related to anastomotic suture methods (including manual suture and stapler anastomosis), gastrointestinal reconstruction methods, and incomplete hemostasis during surgery $[17,18]$. There are few studies on the relationship between neoadjuvant chemotherapy and anastomotic bleeding. There is no clear mechanism to clarify the relationship between the two. However, as far as this study is concerned, neoadjuvant chemotherapy has no obvious effect on anastomotic bleeding. The hematological toxicity of neoadjuvant chemotherapy may lead to a decrease in platelets in the blood, a decrease in certain coagulation factors, and an increased risk of bleeding $[19,20]$, however, these mechanisms of action may have minimal effects compared with surgical methods and intraoperative hemostasis. Due to the small number of bleeding samples included in this study, the results may be biased, and the samples need to be further expanded for exploration.

Neoadjuvant chemotherapy is an effective treatment method that reduces tumor staging, improves surgical resection rate, and prolongs the survival time of patients. Anastomotic complication is a serious postoperative complication that cannot be completely avoided.

Citation: Chuanxu L, Zengwu Y, Mi J, Bin Y, Hongming C, et al. (2021) Influence of Neoadjuvant Chemotherapy on Anastomotic Complications and Long-Term Prognosis after Laparoscopic Radical Gastric Cancer Surgery. J Clin Case Stu 6(4): dx.doi.org/10.16966/24714925.232 
Although neoadjuvant chemotherapy and anastomotic complications may have some potential mechanisms of action, neoadjuvant chemotherapy is not an independent risk factor for anastomotic complications.

\section{Fund Program}

Research Project of Shandong University (3460019005); Yantai Science and Technology Development Plan (2019MSGY136).

\section{References}

1. Nagasako Y, Satoh S, Isogaki J, Inaba K, Taniguchi K, et al. (2012) Impact of anastomotic complications on outcome after laparoscopic gastrectomy for early gastric cancer. Br J Surg 99: 849-854.

2. Jiang L, Yang KH, Guan QL, Cao N, Chen Y, et al. (2013) Laparoscopyassisted gastrectomy versus open gastrectomy for resectable gastric cancer: an update meta-analysis based on randomized controlled trials. Surg Endosc 27: 2466-2480.

3. Lee WL, Chan IS, Wang PH (2020) Does a simple hematological examination predict the response and side effects in patients undergoing induction chemotherapy and/or neoadjuvant chemotherapy? J Chin Med Assoc 83: 107-108.

4. Bray F, Ferlay J, Soerjomataram I, Siegel RL, Torre LA, et al. (2018) Global cancer statistics 2018: GLOBOCAN estimates of incidence and mortality worldwide for 36 cancers in 185 countries. CA Cancer J Clin 68: 394-424.

5. Charruf AZ, Ramos MFKP, Pereira MA, Dias AR, de Castria TB, et al. (2020) Impact of neoadjuvant chemotherapy on surgical and pathological results of gastric cancer patients: A case-control study. J Surg Oncol 121: 833-839.

6. Aurello P, Cinquepalmi M, Petrucciani N, Moschetta G, Antolino L, et al. (2020) Impact of Anastomotic Leakage on Overall and Diseasefree Survival After Surgery for Gastric Carcinoma: A Systematic Review. Anticancer Res 40: 619-624.

7. Palmer $P$, Egger $M$, Philips $P$, McMasters $K M$, Scoggins $C R$, et al. (2020) Predictive preoperative and intraoperative factors of anastomotic leak in gastrectomy patients. Am J Surg 220: 376-380.

8. Makuuchi R, Irino T, Tanizawa Y, Bando E, Kawamura T, et al. (2019) Esophagojejunal anastomotic leakage following gastrectomy for gastric cancer. Surg Today 49: 187-196.

9. Kamarajah SK, Navidi M, Griffin SM, Phillips AW (2020) Impact of anastomotic leak on long-term survival in patients undergoing gastrectomy for gastric cancer. Br J Surg 107: 1648-1658.
10. Nakagawa A, Fujimoto $H$, Nagashima $T$, Sangai $T$, Takada $M$, et al. (2020) Histological features of skin and subcutaneous tissue in patients with breast cancer who have received neoadjuvant chemotherapy and their relationship to post-treatment edema. Breast Cancer 27: 77-84.

11. Jeswani G, Paul SD, Jha AK (2018) Advances in the Delivery of Cancer Therapeutics: A Comprehensive Review. Curr Drug Deliv 15: 21-36.

12. Haskins IN, Kroh MD, Amdur RL, Ponksy JL, Rodriguez JH, et al. (2017) The Effect of Neoadjuvant Chemoradiation on Anastomotic Leak and Additional 30-Day Morbidity and Mortality in Patients Undergoing Total Gastrectomy for Gastric Cancer. J Gastrointest Surg 21: 1577-1583.

13. Ikoma N, Das P, Blum M, Estrella JS, Devine CE, et al. (2017) Preoperative Chemoradiation Therapy Does Not Increase Risk of Anastomotic Leak in Patients With Gastric Cancer. Int J Radiat Oncol Biol Phys 99: 660-666.

14. Maggiori L, Bretagnol F, Aslam MI, Guedj N, Zappa M, et al. (2014) Does pathologic response of rectal cancer influence postoperative morbidity after neoadjuvant radiochemotherapy and total mesorectal excision? Surgery 155: 468-475.

15. Zhu DS, Cao JW, Geng MF, Huang XY, Hu CM, et al. (2020) Wide Gastric Conduit Increases the Risk of Benign Anastomotic Stricture After Esophagectomy. Am Surg 86: 621-627.

16. Deng KF, Liu QX, Zhou D, Min JX, Dai JG (2015) Hand-sewn vs linearly stapled esophagogastric anastomosis for esophageal cancer: a meta-analysis. World J Gastroenterol 21: 4757-4564.

17. Kim KH, Kim MC, Jung GJ, Jang JS, Choi SR (2012) Endoscopic treatment and risk factors of postoperative anastomotic bleeding after gastrectomy for gastric cancer. Int J Surg 10: 593-597.

18. Park JY, Kim YW, Eom BW, Yoon HM, Lee JH, et al. (2014) Unique patterns and proper management of post gastrectomy bleeding in patients with gastric cancer. Surgery 155: 1023-1029.

19. Qi L, Luo Q, Zhang Y, Jia F, Zhao Y, et al. (2019) Advances in Toxicological Research of the Anticancer Drug Cisplatin. Chem Res Toxicol 32: 1469-1486.

20. Babaie D, Shamsian BS, Momtazmanesh N, Godarzipour $H$ Amirmoini M, et al. (2019) Rapid Desensitization for Hypersensitivity Reactions to Chemotherapeutic Drugs; A Case Series. Iran J Pharm Res 18: 1047-1051. 